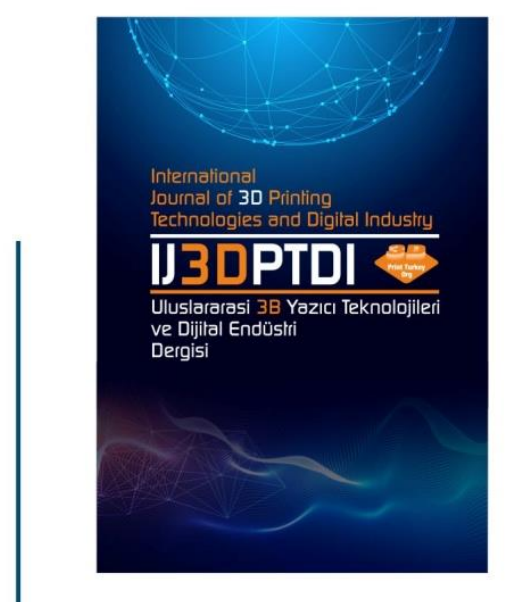

ULUSLARARASI 3B YAZICI TEKNOLOJILERI

VE DIJITAL ENDÜSTRI DERGISI

INTERNATIONAL JOURNAL OF 30 PRINTING TECHNQLOGIES AND DIGITAL INDUSTRY

I55N:2602-3350 [Online]

URL: https://dergipark.org.tr/ij3dptdi

\title{
SYSTEM OF INTELLIGENT MEASUREMENT AND CONTROL FOR THE CONDITION OF MOVING OBJECTS
}

Yazarlar (Authors): Lala Bakirova (1)Aladdin Bayramov (1D*

Bu makaleye şu şekilde atıfta bulunabilirsiniz (To cite to this article): Bakirova L., Bayramov A., "System Of Intelligent Measurement And Control For The Condition Of Moving Objects" Int. J. of 3D Printing Tech. Dig. Ind., 5(3): 426-434, (2021). 


\title{
SYSTEM OF INTELLIGENT MEASUREMENT AND CONTROL FOR THE CONDITION OF MOVING OBJECTS
}

\author{
Lala Bakirova $^{\mathrm{a}}$ (iD) Aladdin Bayramov ${ }^{\text {(iD) }}$ * \\ ${ }^{a}$ Azerbaijan State Oil and Industry University, Information technologies and management, Instrument engineering \\ Department, AZERBAIJAN \\ ${ }^{b}$ Azerbaijan Railways CJSC, Department of İnfrastructure, Head of signaling and communication service, \\ AZERBAIJAN \\ ${ }^{*}$ Corresponding Author: bayramov.aladdin@inbox.ru
}

(Received: 12.09.2021; Revised: 29.10.2021; Accepted: 07.11.2021)

\begin{abstract}
An intelligent system that monitors the speed and condition of a moving object is designed to reduce the risk of potential threats that may arise with autonomous decision-making capabilities depending on the situation and exchange information with databases via remote network sensors. Risk assessment based on information obtained from stationary metering stations installed at intersections and control crossings, object characteristics parameters and side-effect parameters, alerting the central management system based on the results, and making decisions on regulating the speed of the facility, depending on the level of danger and whether the decision is approved by the center, providing information about the facility on the board, sending alerts to security systems at intersections, and identifying opportunities for change by influencing decisions based on risks The characteristic technical parameters of the inflated object depend on internal and external factors and making appropriate decisions according to the risk assessment, etc. information on processes, monitoring, and control. Monitoring of working conditions with the participation of the remote control wireless sensor network in the central control room.
\end{abstract}

Keywords: Control System. Remote Measurement. Remote Control. Wireless Communication. Intelligent System. Diagnostics. Monitoring. Intelligent Sensors.

\section{INTRODUCTION}

The use of a data source appropriate to the facility and the receiver unit serves as a standard structural solution for remote measurement and control systems. Only the methods and means of obtaining information differ from different structural solutions. It means the use of human and unmanned aerial vehicles to monitor the condition of an object, collect information, observe, analyze, evaluate and conduct certain research at a distance to predict the effectiveness of control.

In this regard, it promotes the development of intelligent remote measurement and control systems with extensive functionality, diagnostics of technical nodes based on the measurement of characteristic parameters to monitor the status of two or more objects and interacting active objects using a local (autonomous) system for each object. The advantages of fully automatic driverless systems in terms of capacity are provided in the new railway systems. In most cases, construction or other costs to increase capacity are very high compared to the cost of an alarm system. With the development of technology, the difference between automatic driving (steering) and mechanical driving alarm systems has decreased to 10$15 \%$. If the capacity of the railway system to be installed is high, mobile section signaling systems should be preferred over fixed sections. The use of open-source systems that meet international standards, such as Eurobalise, Global System for Mobile Communications - Railway (GSM-R), Communications-Based 
Train Control (CBTC), will facilitate future extensions, manage different trains on the same train, and so on. It is important in terms of advantages [1].

a new standard recognized as the European Railway Traffic Control System (ERTCS), the movement of two consecutive trains as a unit on a single railway line was modeled using a hybrid technique known as the Batches Petri Nets. The main advantage of using BPN is to provide both separate and continuous operations in train control systems. This allows for easy tracking of train movements. In this case, with the use of various controller architectures, it is planned to construct a development schedule for BPNs, diagnose failures and develop the rules for recovery of failures [2]. Improving the reliability of operating technical equipment is one of the most important conditions for the efficient and safe operation of railway transport [3]. In this regard, one of the main conditions for the construction of a risk and reliability analysis control system is the development of a system of operational reliability indicators, methods of their calculation, etc. [4].

The development of the train protection system consists of several stages, where the analysis of external influences when receiving codes from the rails shows that the code combinations of locomotive receivers can be subjected to the influences of internal and external factors in the process of transmitting code signals to railways. Impulse and harmonic obstacles are more common. Therefore, they can reflect themselves in the negative effects of reverse traction current and locomotive power plants, as well as uneven residual magnetization on the rails, operating rails, and rails placed on the side of the road. External influences, such as a false indication of the short-term locomotive traffic lights, inconsistency of the data transmitted to the locomotive, and the indicator of the traffic light, can lead to malfunctions of the automatic locomotive signaling system. In real conditions, there are obstacles that practically lead to code distortion, and if they are within the available limit, normal transmission is not violated [5].

The (Continuous automatic locomotive signaling) ALSN code system is commonly used on railways with a rail distance of $1520 \mathrm{~mm}$. In this case, the control system receives the following code signals: G-green, Yyellow, R-red, YG-green-yellow. Here, a global positioning system (GPS) is offered for determining the coordinates of objects to monitor the movement of trains. In this case, the locomotive control modules transmit information directly to the locomotive control system by controlling the change of the profile and coordinates of the road. Regardless of the state of the locomotive controllers, the change in the coordinates of the road profile is evaluated, and in this way, controlling the speed of the locomotive is carried out [6].

Automatic code installations are connected to the rail circuits between the traffic lights and the information is transmitted to the train in the form of a code. A specific code combination has been adopted for each traffic light indicator, and it corresponds to the upcoming traffic light indicator. In the three-digit signaling system, if the upcoming traffic light is red, a yellow-red code is transmitted to the train, if it is yellow, a yellow code, if it is green, a green code is transmitted to the train. The codes used in the (Continuous automatic locomotive signaling) ALSN system are used to check the vigilance of drivers during train movement, speed limit, and braking requirements, speed and driver's movement, and the data is transmitted from the road signaling equipment to the locomotive [7].

Thanks to the international and domestic importance of railway transport, modern railway communications with modern bridges, tunnels, and stations have been established. Ensuring a high level of efficiency and safety on the railway is carried out by modern control and diagnostic systems. However, freight and passenger train accidents continue. Research shows that it is possible to improve the safety of railway transport by solving certain problems, such as ensuring control over the latent period of the technical condition of railway rails, railway bridges, tunnels, crossings, individual wagons, and all personnel [8].

In this paper, APNs are used to design railway interlocking and signalization systems for a sample railway yard. APNs are one of the most efficient methods for designing discrete event control systems. With the help of token passing logic, the obtained APN model can be easily implemented on a PLC by converting it into 
ladder diagrams. The main advantage of using APN is that it includes both actuators and sensors. With less effort, we can trace the errors easily as the graphical illustrations are simple [9].

As a substitute for the human mind in a sense, intelligent systems differ by being able to make operational decisions for control with such types as approaching complex problems and their solutions, obtaining a set of data on variable time, space, and internal and external factors from sensors distributed to different measurement areas, and on their basis, distributing hierarchical and complex control. In this case, along with obtaining information, the exchange of information between different levels, use of special processing, structural and software solution methods for determining and increasing the correctness of the information obtained are among the issues to be addressed. For this reason, in the development of intelligent systems, the development of solution algorithms allowing to make right decisions based on the principle of IF THEN in the possibility of different solutions, the creation of an exemplary, accurate data bank for making the right decisions and the comparison of measurement and control results with those data, making the right decisions as a result of analysis are of particular importance [10].

\section{THE PROBLEM STATEMENT AND THE PURPOSE OF THE WORK}

Since distortion of codes in the code system presented as a solution in existing sources does not fully guarantee traffic safety, the development of an intelligent remote measurement and control system with a wide range of functional capabilities, consisting of a local (autonomous) system for each facility, having the ability to promptly perform the diagnosis of technical nodes and related problems by measuring characteristic parameters with distributed sensors to monitor the condition of two or more facilities and interacting active facilities, is a topical issue.

The proposed intelligent system is intended to function as an intelligent system having such functional capabilities of performing measurement and control operations to control the parameters of external factors, operational processing and analysis, having high reliability and flexibility, availability of a database which collects parameters and additions are made in during each movement of the facility, determining the position of the facility on board and its location relative to other facilities, information exchange with on-board systems and the center of other moving facilities, self-diagnostics, self-correction, making independent decisions to change the state of the facility identifying traffic safety along with the technical state of the facility itself.

\section{METHODS AND MATERIAL}

Active objects moving in the same line (Object 1) can detect any fault on the road, i.e. on the rail lines (e.g. point A) and transmit information about this fault to the objects following it. It has the ability to transmit information transmitted to the onboard control system of the object to the central control room via a network of optical cables and intelligent sensors. According to the transmitted information, the movement of active objects in the area is restricted, the speed limit is lowered or the hazardous area is closed for traffic until the fault is eliminated.

Based on the results of the two measurements, it is intended to obtain accurate information and make an objective decision. According to levels, the integrity of signals received from the rails is monitored using a transmitter-receiver connected to the rails and intelligent sensors placed on board of the object and allowing remote measurements. Each transmitter-receiver device amplifies the signal it receives to a certain extent and transmits it to its predecessor. The executive body works at the appropriate values of the signal levels. If the signal levels do not meet the standards, the executive body will not receive the appropriate signal, which will indicate a fault on the road. It is also planned to transmit this information via a closed circuit to the center, to a system of the same rank installed in other objects moving in the same and opposite directions. The following are designed to ensure traffic safety: 
- $\quad$ Placement of sensors for safety-on the board of the moving object and in the dispatching points and death knots, which can be placed at regulated and unregulated crossroads;

- $\quad$ Detection of defects between on-board measurement and control systems of objects moving in the same and opposite directions; exchange of information related to other hazards and technical conditions;

- Creation and regular updating of the database following the characteristic parameters of the technical indicators of the object and internal and external influencing factors;

- $\quad$ Possibility of prioritizing autonomous decision-making in the event that the central control base is unable to make a decision in the event of hazard detection;

- $\quad$ To create rail defects, contact and contactless measurement methods and tools are used.

- Interactive communication with geographic information systems reflecting the coordinates and state of regulated and unregulated road crossings in the direction of traffic

- $\quad$ Automatic barrier installation and control at regulated and unregulated railway crossings;

- $\quad$ Adjustment of movement speed depending on the level of hazard and risk.

- Installation of SCADA virtual system of intelligent on-board system.

The advantage of the intelligent system is that the on-board systems of the train and other objects moving in the same and opposite directions constantly transmit the information received from several channels by providing the required speed based on the instructions of the relevant database. It also consists of a continuous comparison of the actual speed with the allowable speed. In industry, there is a great demand for the state of the object and the control of technical equipment, depending on the requirements for the monitoring of vehicles by making decisions based on the results obtained by observing the state of objects measurement, control, and comparison of parameters according to the situation.

In this case, various defects in the rail circuit weaken the conductivity of the rail, and the transmission of the necessary code signals reflecting the situation to the onboard system is limited. This leads to the loss of information about the corresponding part of the rail and the malfunction that has arisen there. In addition to the state of the object, it is possible to monitor its location using satellite communication using GSM, GPRS modules, and sensors installed in local radio towers (active and passive). Various types of sensors can be used to measure these parameters. Both objects are being watched. It is possible to manage objects both locally and remotely.

The more data, the higher the accuracy of the information. The accuracy of the information is the quality of the information. In the new structure to be applied, the braking distance is determined not only by the indicators of traffic lights but also by the gap of the unit areas to be installed. DPT-data processing and transmission, RWTRT-radio wave transmission and reception tower, RF-road facilities, RL-rail line, RCrail circuit, AM-axis meter, G-generator.

Only 3 code combinations are considered here, i.e. codes are sent via wireless radio waves, which mean green, yellow, red, or red-yellow signs. According to the color of each traffic light and, in addition, depending on the condition of the road ahead (whether the road is busy or empty), signals are transmitted to the trains via rails, sensors, and radio waves. The traffic light indicator is first transmitted to the DPT equipment, and this device also sends a signal to the train about the accuracy of the information after comparing the gap or busy of the upcoming block areas via the traffic light indicator. The transmission length of the signals is equal to the lengths of the block fields. The codes are constantly being compared with each other. The codes corresponding to the traffic light indicators are transmitted by rail. In a computerbased centralization and interlocking system, the wireless signal checks the gap between the block areas with both the traffic light and the oncoming rail circuit or axis meter sensor and, after making a decision, it sends the signals from the RWTRT to the train. The train devices continuously receive codes from rails and radio signals, while continuing to move in a towed system by receiving data from smart sensors installed on the track. If moving objects with the same status are moving on the same line, and if there is a problem with one of the objects, the objects send information to each other, and the center. 
Objects moving on the line with the same status can receive information from passive sensors, static objects installed on the edge of the line, obtaining information about the coordinates of other objects they provide information about their location relative to them. Thus, the created intelligent system performs the following functions: execution of measurement algorithm and obtaining measurement results, information processing, calibration of the measurement channel, linearization of the conversion characteristics of the measurement channels, scaling, calculation of measurement errors, and presentation with measurement results, measurement channel correction, measurement statistical processing of results, etc.

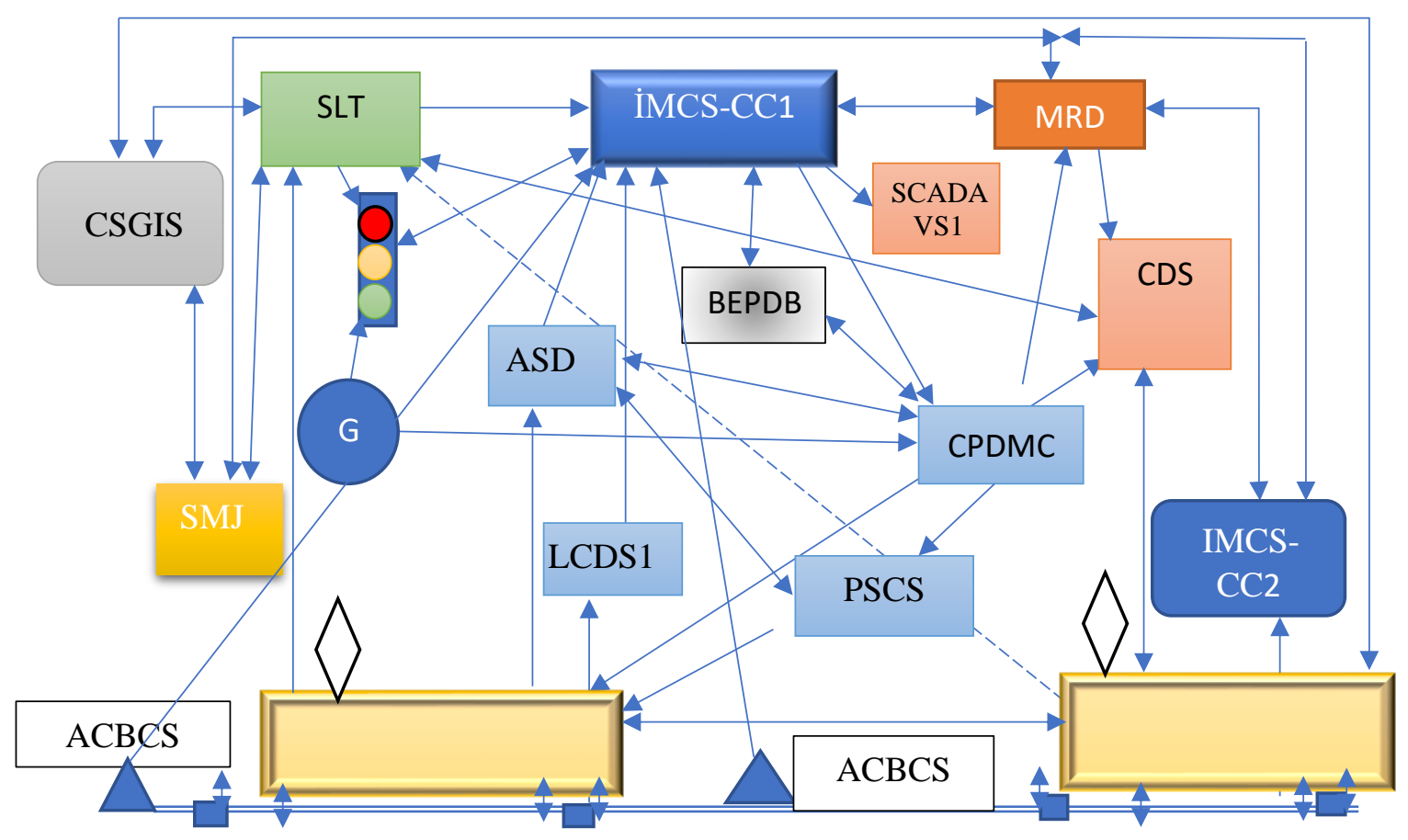

Figure 1. Intelligent control system for the speed and state of trains.

TL-traffic light, SLT-system for locating trains, DPT-data processing and transmission, G-generator, FRASdevice for recording the actual speed, RTRC-radio wave transmission and reception center, SMJ-stationary measuring junction, SCD-Speed Comparison Device, LCDS-line condition diagnostic system, PSCSpermitted speed control system, GIS CS-geographic information system communication scheme, ACBCSautomatic crossing barrier control system, IMCS-CC1-information measurement control system-central controller, MRD-measuring receiving device, CDS-central dispatching station, BEPDB-basic and effective parameter data bank; CPDMC-comparison and processing decision-making controllers.

With the help of the system, it is possible to ensure the protection of objects of the same status, moving consistently on certain predetermined coordinates, safety, continuous control of speed limits, dependent and independent decision-making, as well as the personal and physical safety of people. In addition, the advantage of the work is that it is possible to implement an intelligent system to know the location of moving objects (trains) and ensure safe movement, using a high-performance element base, and expand software using hardware. It is intended to further enhance the functionality by using it as a mobile add-on module. The capabilities are applied to the system by downloading additional relevant software. 


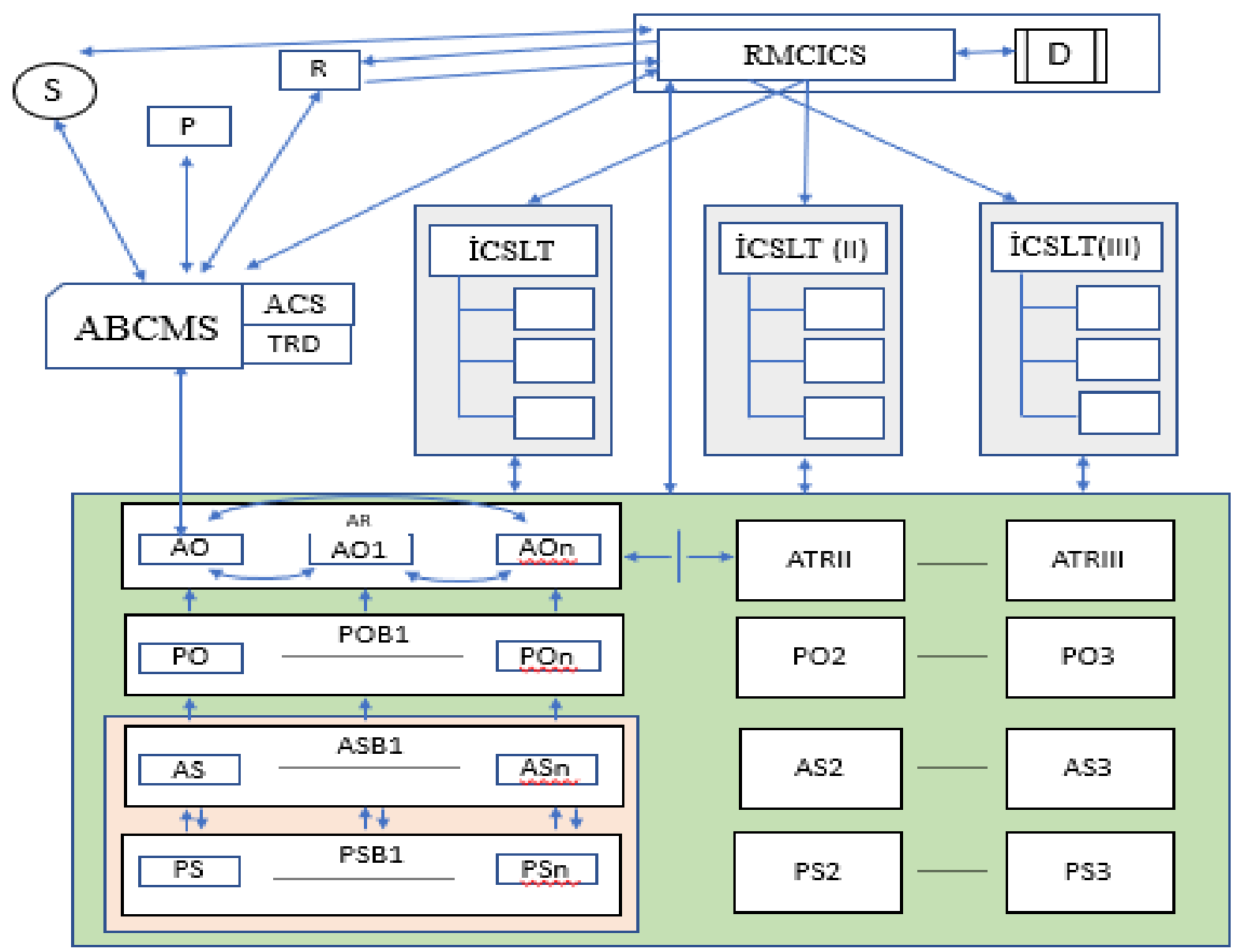

Figure 2. The remote and local intelligent control system of traffic

AOBCMS-autonomous on-board control and measurement system, ACS-autonomous control system, TRDtransmitter-receiver device, S-satellite, P-physical, R-remote, ATR-active traffic route, AO-active object, PO-passive object, AS-active sensor, PS-passive sensor, RMCICS-remote movement control and intelligent control system, D-dispatcher, ICSLT-intelligent control system of local traffic.

This article provides a brief overview of the signal systems used in railway systems and the advantages of automatic train operation in today's technology, where signaling and communication have made great advances. The system, which is located in objects moving in the same and opposite directions, can be used as an autonomous system that allows trains of the same status to receive information from static objects (including death knots that can be placed at dispatching points and regulated and unregulated intersections), know the location of an object it is placed and the location of another object. Thus, active objects in motion have control functions by exchanging information, and at the same time ensure the security of other moving objects. The information obtained in this case is stored in memory in the onboard system of the carrier and transmitted to the central control system at certain time intervals, at the request of the ground center. It has the ability to interfere with the processes taking place on the object.

According to the practical construction and repair requirements, it is determined for the system design of typical projects, and according to these new requirements, the following is taken into account in the new signal system: 
The number of trackside equipment in the new alarm system should be reduced. The new alarm system adopts a virtual unit or moving unit to replace the fixed unit system. The unit section can be flexibly adjusted according to the volume of traffic to improve traffic capacity. By optimizing the system structure, the new alarm system should minimize lifetime costs (i.e., construction, placement, and maintenance costs) as much as possible. The system structure, logic model, and standard interface module are designed to meet the special needs of different railway crossings.

Autonomous localization and checking the integrity of trains is carried out under the onboard control of the vehicle. The system should have a modular principle. High carrying capacity is achieved by optimizing the control mode for trains. An intelligent system that allows to safely control objects by determining their state has the functions of an intelligent system that allows to determine the situation between two devices (trains) of the same status, ensure security, and make independent decisions based on the security of the mutual situation, in contrast to the existing systems of control of facilities by different means from the central control room. Using Arduino uno with low parameters in the existing laboratory conditions, the contact distance of autonomous objects in the system was taken to be $1 \mathrm{~km}$. Ensuring security by increasing this distance and arranging a secure information exchange can be a topic of discussion. The system is based on two modules and is practically operational.

The simplicity of the program allows the use of small-capacity memory. However, with the expansion of technical capabilities, it is possible to significantly increase this distance (5 km, up to $10 \mathrm{~km}$ and more) using Laura modes. It should be noted that the use of the system in objects of a special category allows you to solve problems such as remote control and management from a central point. In this sense, the autonomous object presents itself as an intellectual system. An autonomous system is able to exchange information, make free decisions, self-diagnostics, self-correction, control of internal parameters, measurement control, logic and control operations, high level of reliability, age and flexibility, and selfprogramming. It is also able to control its own position and location relative to other objects. has the functions of an intelligent system, such as managing the status of an incoming object, expressing its position to other objects of the same status.

The discussion in this paper focuses on technical systems only. A technical system is a product developed by a supplier that includes its design, implementation, and support documentation. It should be noted that:

- The development of a technical system starts with its system requirements specification and ends with its safety approval.

- Human operators and their actions are not included in a technical system.

- Maintenance is not included in the definition, but maintenance manuals are.

- Technical systems can be subject to a generic type of approval for which a stand-alone risk acceptance criterion is useful.

\section{RESULT AND DISCUSSION}

Advanced intelligent control is mainly used in comprehensive application scenarios such as computer technology, GPS positioning technology, LORA, NRF modules or precision sensing technology. With the increasingly fierce competition in the product market, intelligent products have obtained good application advantages in practical operations and applications. The main results include greatly improving the operator's operating efficiency, improving the quality of work in some dangerous places, and reducing work intensity, solving application of some dangerous and key constructions jobs, enhanced machine automation and intelligence, improved equipment reliability and reduced maintenance costs, and intelligent fault diagnosis, environmental protection and energy saving, etc.

Other intelligent control methods such as machine learning control, expert system and genetic algorithm control, etc., or the aforementioned artificial neural networks and fuzzy control, will be from communication 
technology, manufacturing technology, construction technology, transportation technology and the integration of energy technology and other different levels is also an important task for us to continue to make good use of the advantages of intelligent control methods and eliminate the disadvantages in the future.

\section{CONCLUSION}

The proposed intelligent measurement and control system allows us to achieve the following topical functions in controlling facilities, provided that traffic safety is increased:

- Controlling various parameters between static, stationary facilities through a system of intelligent, remote-controlled sensors;

- Remote control or continuous exchange of information between facilities (trains) moving in the same and opposite directions with the same status;

- Diagnosis of traffic lines and technical devices of the facility by means of remote and non-contact tools installed directly on the facility;

- Creation of a database of technical parameters and indicators of influencing factors;

- Operational on-board processing and comparative analysis of information;

- Self-government, self-correction, and autonomous decision-making;

- Interactive communication with geographic information systems reflecting the coordinates and state of regulated and unregulated road crossings in the direction of traffic;

- Arrangement and control of automatic barriers at regulated and unregulated railway crossings;

- Regularization of speed rate depending on the level of danger and risk;

- Installation of the SCADA virtual system of intelligent on-board system;

- Minimizing the interval and increasing the capacity of the safe traffic;

In this case, it is possible to ensure security on the base of the exchange of information between facilities (trains), as well as between other moving facilities whose movement intersects with them at the same level. The development of the system that allows them to be protected and controlled by determining the state of the facility is intended by taking into account such key issues as the development of two-way intelligent remote measurement and control systems with the use of modern intelligent sensors, adaptive iteration methods, error correction, partial and, in some cases, independent control functions, self-studying, and linearization. Intelligent system self-diagnostics, determining location, cause of errors, program error detection, detection and elimination of external influences on signals received from sensors and important for control, automatic checking and self-calibration of the measuring channel, determining the probability of failure of elements and forecasting the prevention of hazardous situations that may occur with the elimination of failures.

According to the changes in the controlled facility and the facilities that interact with it, intelligent control is useful for improving the security and reliability of automated systems. The above-mentioned functionality is of great importance to prevent hazardous situations that may occur in the event of disconnection from the center.

\section{REFERENCES}

1. Söyler H, Açıkbaş S. "Raylı Toplu Taşımda Sinyalizasyon Sistemleri”, Cilt 9, Sayı 3, Sayfalar 1 - 14, 2017.

2. Durmuş M.S., Yıldırım U., Söyleme M.T., "Interlocking System Design for ERTMS/ETCS: An Approach with Batches of Petri Nets”,Vol. 45, Issue 29, pp 110-115, 2012.

3. Gapanovich V.A. "Ensuring the reliability of technical means/V.A. Gapanovich // Railway transport", No 9. - p. 2 $5,2008$.

4. Gapanovih V.A. "Development and implementation of URRAN technology in railway transport", Vol. 4, pp. 3-10, 2013. 
5. Pultyakov A. V., Skorobogatov M. E., "System analysis of the sustainability of automatic locomotive signaling systems", Received: February 12, 2018. Modern technologies. System analysis. Modeling, Vol. 57, no. 1, 2018.

6. Liudvinaviius L., , Dailydka S., and Sadkowski A. "New Railway Traffic Control System Possibilities", Vol. 11, Issue 11, 2016.

7. Baluev N., Shamanov V.I, "Rationing Of Resistance Of Elements Of The Traction Rail Network", Automation, Communication, Informatics, pp. 13-18, 2014.

8. Liudvinaviius L., and Sadkowski A. "Train Protection Systems On Different Railways", Springer International Publishing AG2017. Rail Transport—Systems Approach (pp.273-318), March 2017.

9. Malakar B., Roy B.K.. "Railway Fail-Safe Signalization And Interlocking Design Based On Automation", ICICES2014-S.A.Engineering College, Chennai, Tamil Nadu, India. February 2015.

10. Xiao L, Li M, Wang F, Li Y. "A Hierarchical Framework For An Intelligent Launch Site System, 2018 IEEE International Conference on Information and Automation (ICIA), Wuyishan, China, pp. 814-818., 2018. 